\title{
Two-site polaron problem : a perturbation approach with variational basis states
}

\author{
A. N. Das] and Jayita Chatterjee] \\ Saha Institute of Nuclear Physics \\ 1/AF Bidhannagar, Calcutta 700064, India
}

PACS No.71.38. +i, 63.20.kr

\footnotetext{
${ }^{1}$ e-mail: atin@cmp.saha.ernet.in

2 e-mail: moon@cmp.saha.ernet.in
} 


\begin{abstract}
A convergent perturbation method using modified Lang Firsov transformation is developed for a two-site single-polaron system. The method is applicable for the entire range of the electron-phonon coupling strength from the antiadiabatic limit to the intermediate region of hopping. The single-electron energies, oscillator wave functions and correlation functions, calculated using this method, are in good agreement with the exact results.
\end{abstract}




\section{Introduction}

The interaction of conduction electrons with lattice vibrations is described by the so called electron-phonon problem. The Holstein model [1] is one of the fundamental models which has been studied widely in this context. The model consists of a one-electron hopping term, Einstein phonons at each site and a site-diagonal interaction term which couples the electron density and ionic displacements at a given site. For weak electron-phonon (e-ph) coupling the frequency of the phonons and the effective mass of the the electron are renormalized, which are described by the Migdal approximation [2]. For large e-ph coupling the electrons are selftrapped in the lattice deformation producing a small polaron. The motion of the electron is then accompanied by the lattice deformation. This results in a large effective mass or reduced effective hopping of the dressed electrons (polarons). The Lang-Firsov (LF) method based on the LF canonical [3] transformation works in this strong coupling region in the antiadiabatic limit. For weak coupling the Migdal approximation is satisfactory. However, no conventional analytical method exists at present which is beleived to describe a Holstein model for the entire range of the coupling strength. So, it would be useful to develop or identify an analytic method which could be applied to both the strong and weak coupling cases. Ranninger and Thibblin [4] made an exact diagonalization study of a two-site polaron problem and showed that the behavior of the polaron differs very much from that predicted by the classical LF method. Marsiglio [5] extended those calculations to the bulk limit in one dimension by studying the Holstein model with one electron up to 16 site lattices. He concluded that for intermediate coupling strength neither the Migdal nor the small-polaron approximation is in quantitative agreement with the exact results. Kabanov and Ray [6] and Alexandrov et al. [7] noted that for $t>\omega_{0}$ the adiabatic small-polaron approximation describes the ground state energy ac- 
curately except for intermediate coupling strength. Ranninger and Thibblin [4] and Marsiglio [5] studied also the correlation functions using exact diagonalization technique with the finite size Holstein model and found that the results are non trivial and cannot be described by any conventional analytical method. Recently, de Mello and Ranninger [8] emphasized this point further.

The objective of the present work is to search for an analytical method which may be applicable reasonably well for the major range of e-ph coupling strength. For our study we consider a two-site one-electron Holstein model for which exact results are available [四]. Previously, we [9], 10] investigated the ground state energy and the nature of polarons in a two-site and a four-site Holstein model using the modified Lang Firsov (modified LF) transformation and two-phonon coherent states and found that the energy obtained within such method is very close to the exact result. In this work we develop a perturbation expansion within the modified LF transformation and show that this expansion converges for the entire range of the coupling strength from the antiadiabatic limit to the intermediate region of hopping. The energy and the correlation functions calculated within our approach are almost identical with the exact results.

\section{Formalism}

The Hamiltonian of a two-site one-electron Holstein model reads as

$$
H=\sum_{i, \sigma} \epsilon n_{i \sigma}-\sum_{\sigma} t\left(c_{1 \sigma}^{\dagger} c_{2 \sigma}+c_{2 \sigma}^{\dagger} c_{1 \sigma}\right)+g \omega_{0} \sum_{i, \sigma} n_{i \sigma}\left(b_{i}+b_{i}^{\dagger}\right)+\omega_{0} \sum_{i} b_{i}^{\dagger} b_{i}
$$

where $i=1$ or 2 , denotes the site. $c_{i \sigma}\left(c_{i \sigma}^{\dagger}\right)$ is the annihilation (creation) operator for the electron with spin $\sigma$ at site $i$ and $n_{i \sigma}\left(=c_{i \sigma}^{\dagger} c_{i \sigma}\right)$ is the corresponding number operator, $g$ denotes the e-ph coupling strength, $b_{i}$ and $b_{i}^{\dagger}$ are the annihilation and creation operators, respectively, for the phonons corresponding to interatomic 
vibrations at site $i, \omega_{0}$ is the phonon frequency. In Hamiltonian (1) there is no spin dependent or spin reversal term so for the study of one-electron case the spin index is redundant. In the following we shall not use the spin index.

Introducing new phonon operators $a=\left(b_{1}+b_{2}\right) / \sqrt{2}$ and $d=\left(b_{1}-b_{2}\right) / \sqrt{2}$ the Hamiltonian is separated into two parts $\left(H=H_{d}+H_{a}\right)$ :

$$
H_{d}=\sum_{i} \epsilon n_{i}-t\left(c_{1}^{\dagger} c_{2}+c_{2}^{\dagger} c_{1}\right)+\omega_{0} g_{+}\left(n_{1}-n_{2}\right)\left(d+d^{\dagger}\right)+\omega_{0} d^{\dagger} d
$$

and

$$
H_{a}=\omega_{0} \tilde{a}^{\dagger} \tilde{a}-\omega_{0} n^{2} g_{+}^{2}
$$

where $g_{+}=g / \sqrt{2}, \tilde{a}=a+n g_{+}$and $\tilde{a}^{\dagger}=a^{\dagger}+n g_{+}$.

$H_{a}$ describes a shifted oscillator which couples only with the total number of electrons $n\left(=n_{1}+n_{2}\right)$, which is a constant of motion. The last term in Eq.(3) represents lowering of energy achieved through the lattice deformations of sites 1 and 2 by the total number of electrons.

$H_{d}$ represents an effective e-ph system where phonons directly couple with the electronic degrees of freedom. $H_{d}$ cannot be solved exactly by any analytical method. We now use the modified LF transformation where the lattice deformations are treated as variational parameters [9, 11, 12]. For the present system,

$$
\tilde{H}_{d}=e^{R} H_{d} e^{-R}
$$

where $R=\lambda\left(n_{1}-n_{2}\right)\left(d^{\dagger}-d\right), \lambda$ is a variational parameter and linearly related to the displacement of the d oscillator.

The transformed Hamiltonian is then obtained as

$$
\begin{aligned}
\tilde{H}_{d} & =\omega_{0} d^{\dagger} d+\sum_{i} \epsilon_{p} n_{i}-t\left[c_{1}^{\dagger} c_{2} \exp \left(2 \lambda\left(\mathrm{d}^{\dagger}-\mathrm{d}\right)\right)\right. \\
& \left.+c_{2}^{\dagger} c_{1} \exp \left(-2 \lambda\left(\mathrm{d}^{\dagger}-\mathrm{d}\right)\right)\right]+\omega_{0}\left(\mathrm{~g}_{+}-\lambda\right)\left(\mathrm{n}_{1}-\mathrm{n}_{2}\right)\left(\mathrm{d}+\mathrm{d}^{\dagger}\right)
\end{aligned}
$$


where

$$
\epsilon_{p}=\epsilon-\omega_{0}\left(2 g_{+}-\lambda\right) \lambda
$$

It may be mentioned that with an ordinary LF transformation, where one chooses a phonon basis of the oscillator with a fixed displacement $\left(\lambda=g_{+}\right.$for this case), one can diagonalize the Hamiltonian in absence of hopping. The hopping term, containing off-diagonal matrix elements in the new phonon basis, may then be treated within the perturbation approach in the strong coupling and antiadiabatic limit [13]. However, in order to develop a perturbation theory to be valid for the entire range of coupling strength one should consider a variational phonon basis such that the major part of the hamiltonian could be diagonalized for different values of the coupling strength. The modified LF transformation, where the phonon basis are formed by the oscillator with variable displacement, would serve this purpose.

For the single polaron problem we choose the basis set (for $\tilde{H}_{d}$ )

$$
\begin{aligned}
& |+, N\rangle=\frac{1}{\sqrt{2}}\left(c_{1}^{\dagger}+c_{2}^{\dagger}\right)|0\rangle_{e}|N\rangle \\
& |-, N\rangle=\frac{1}{\sqrt{2}}\left(c_{1}^{\dagger}-c_{2}^{\dagger}\right)|0\rangle_{e}|N\rangle
\end{aligned}
$$

where $|+\rangle$ and $|-\rangle$ are the bonding and antibonding electronic states and $|N\rangle$ denotes the $N$ th excited oscillator state.

Note that the last term in Eq.(5) has only off-diagonal matrix elements connecting bonding and antibonding states with the change in phonon number by $\pm 1$

$$
\begin{array}{r}
\left\langle M, \pm\left|\omega_{0}\left(g_{+}-\lambda\right)\left(n_{1}-n_{2}\right)\left(d+d^{\dagger}\right)\right| \mp, N\right\rangle \\
=\left(\sqrt{N} \delta_{M, N-1}+\sqrt{N+1} \delta_{M, N+1}\right) \omega_{0}\left(g_{+}-\lambda\right)
\end{array}
$$


while the hopping term $H_{t}=-t\left[c_{1}^{\dagger} c_{2} \exp \left(2 \lambda\left(\mathrm{d}^{\dagger}-\mathrm{d}\right)\right)+\mathrm{c}_{2}^{\dagger} \mathrm{c}_{1} \exp \left(-2 \lambda\left(\mathrm{d}^{\dagger}-\mathrm{d}\right)\right)\right]$ has both diagonal and off-diagonal elements in the chosen basis. The diagonal part of $H_{t}$ is given by,

$$
\left\langle N, \pm\left|H_{t}\right| \pm, N\right\rangle=\mp t_{e} \sum_{i=0}^{N}\left[\frac{(2 \lambda)^{2 i}}{i !}(-1)^{i} N_{C_{i}}\right]
$$

where $t_{e}=t \exp \left(-2 \lambda^{2}\right)$ and $N_{C_{i}}=\frac{N !}{i !(N-i) !}$.

The diagonal part of the Hamiltonian $\tilde{H}_{d}$ (in the chosen basis) is considered as the unperturbed Hamiltonian $\left(H_{0}\right)$ and the remaining part of the Hamiltonian $H_{1}=\tilde{H}_{d}-H_{0}$ is treated as a perturbation.

The unperturbed energy of the state $| \pm, N\rangle$ is given by

$$
E_{ \pm, N}^{(0)}=\left\langle N, \pm\left|H_{0}\right| \pm, N\right\rangle=N \omega_{0}+\epsilon_{p} \mp t_{e}\left[\sum_{i=0}^{N}\left(\frac{(2 \lambda)^{2 i}}{i !}(-1)^{i} N_{C_{i}}\right]\right.
$$

The general off-diagonal matrix elements of $H_{1}$ between the two states $| \pm, N\rangle$ and $| \pm, M\rangle$ are calculated as (for $(N-M)>0$ )

$$
\begin{aligned}
& \left\langle N, \pm\left|H_{1}\right| \pm, M\right\rangle=P(N, M) \text { for even }(\mathrm{N}-\mathrm{M}) \\
& \left\langle N, \pm\left|H_{1}\right| \mp, M\right\rangle=P(N, M)+\sqrt{N} \omega_{0}\left(g_{+}-\lambda\right) \delta_{N, M+1} \text { for odd }(\mathrm{N}-\mathrm{M}) .
\end{aligned}
$$

where

$$
\begin{aligned}
P(N, M)= & \mp t_{e}(2 \lambda)^{N-M} \sqrt{\frac{N !}{M !}}\left[\frac{1}{(N-M) !}+\sum_{R=1}^{M}\left[(-1)^{R}\right.\right. \\
& \left.\left.\frac{(2 \lambda)^{2 R}}{(N-M+R) ! R !} M(M-1) \ldots(M-R+1)\right]\right]
\end{aligned}
$$

In the following we present the perturbation corrections to the energies and the correlation functions for the ground and the first excited state. 


\section{The energies and the correlation functions}

\section{A. Ground state:}

For the system considered, the state $|+\rangle|0\rangle$ has the lowest unperturbed energy, $E_{0}^{(0)}=\epsilon_{p}-t_{e}$. The matrix element connecting this ground state and an excited state $|e, N\rangle$ is given by

$$
\begin{aligned}
\left\langle N, e\left|H_{1}\right|+, 0\right\rangle & =\left[-t_{e} \frac{(2 \lambda)^{N}}{\sqrt{N !}}+\omega_{0}\left(g_{+}-\lambda\right) \delta_{N, 1}\right] \delta_{e,-} \quad \text { for odd } \mathrm{N} \\
& =\left[-t_{e} \frac{(2 \lambda)^{N}}{\sqrt{N !}}\right] \delta_{e,+} \text { for even } \mathrm{N}
\end{aligned}
$$

The first order correction to the ground state wave function is obtained as,

$$
\begin{aligned}
\left|\psi_{0}^{(1)}\right\rangle & =-\frac{\omega_{0}\left(g_{+}-\lambda\right)-2 \lambda t_{e}}{\left[\omega_{0}+2 t_{e}\left(1-2 \lambda^{2}\right)\right]}|-, 1\rangle+\sum_{N=2,4, . .} \frac{t_{e}(2 \lambda)^{N}}{\sqrt{N !}\left(E_{+, N}^{(0)}-E_{0}^{(0)}\right)}|+, N\rangle \\
& +\sum_{N=3,5, . .} \frac{t_{e}(2 \lambda)^{N}}{\sqrt{N !}\left(E_{-, N}^{(0)}-E_{0}^{(0)}\right)}|-, N\rangle
\end{aligned}
$$

where $E_{ \pm, N}^{(0)}$ is the unperturbed energy of the state $| \pm, N\rangle$ as given in Eq.(10).

The second order correction to the ground state energy is given by

$$
E_{0}^{(2)}=-\frac{t_{e}^{2}\left(2 \lambda-\frac{\omega_{0}}{t_{e}}\left(g_{+}-\lambda\right)\right)^{2}}{\left[\omega_{0}+2 t_{e}\left(1-2 \lambda^{2}\right)\right]}-\sum_{N=2}^{\infty} \frac{t_{e}^{2}(2 \lambda)^{2 N}}{N !\left(E_{e, N}^{(0)}-E_{0}^{(0)}\right)}
$$

where $\mathrm{e}=+$ or - for even and odd $\mathrm{N}$, respectively.

Now, one has to make a proper choice of $\lambda$, hence choice for the displaced phonon basis, so that the perturbative expansion becomes convergent. In the usual modified LF method $\lambda$ is found out by minimizing the ground state energy of the system. Here we adopt that method and check whether it gives satisfactory results. From our previous studies [9, 10, 11] we know that $\lambda$ remains small as long as $g_{+}<1$, while for large values of $g_{+}$(in the strong coupling limit) it approaches or 
attains the full LF value of $g_{+}$(see Table I). For small values of $\lambda$ the perturbation series involving linear Frohlich type (polaron-phonon) interaction term $\left(\propto\left(g_{+}-\lambda\right)\right)$ converges automatically, while that involving hopping, containing powers of $2 \lambda$, would converge provided $t<\omega_{0}$ or $t \sim \omega_{0}$. For strong coupling, as $\lambda$ approaches to $g_{+}$, the Frohlich (polaron-phonon) interaction term almost vanishes as well as $t_{e}$ becomes very small so the perturbation series converges. Thus, it is expected that the perturbation method following the modified LF transformation would work satisfactorily in both the weak and strong coupling limits. In this work we have shown that it works reasonably well for whole range of the coupling strength for $t \leq \omega_{0}$

Following the spirit of the modified LF method the value of $\lambda$ is found out as $\lambda=\omega_{0} g_{+} /\left(\omega_{0}+2 t_{e}\right)$ from minimization of the unperturbed ground state energy. It is interesting to note that for this particular choice of $\lambda$, the coefficient of $|-, 1\rangle$ in Eq. (14) as well as the first term in the r. h. s. of Eq. (15) vanishes. In other words, the off-diagonal matrix element between the states $|+, 0\rangle$ and $|-, 1\rangle$ becomes zero for the modified LF choice of phonon basis which leads to small perturbation correction to the ground state within this method.

To check whether the perturbation series is converging properly we have calculated and computed the third order correction to the energy for the ground state. The third order correction $\left(E_{0}^{(3)}\right)$ to the ground state energy is given by,

$$
E_{0}^{(3)}=\sum_{k \neq 0}\left[\left(H_{1}\right)_{0 k} \sum_{m \neq 0}\left[\frac{\left(H_{1}\right)_{k m}\left(H_{1}\right)_{m 0}}{\left(E_{0}^{(0)}-E_{k}^{(0)}\right)\left(E_{0}^{(0)}-E_{m}^{(0)}\right)}\right]\right]
$$

where the subscript $\mathrm{k}, \mathrm{m}$ denote the states $| \pm, N\rangle$ with the unperturbed energy $E_{ \pm, N}^{(0)}$ and the subscript 0 refers to the ground state $|+, 0\rangle$. The off-diagonal matrix elements of $H_{1}$ are calculated using Eqs.(11) and (12).

The ground state wave function of $\tilde{H}_{d}$ considering up to the second order cor- 
rections in perturbation is given by,

$$
|\tilde{G}\rangle \equiv|+, 0\rangle+\left|\psi_{0}^{(1)}\right\rangle+\left|\psi_{0}^{(2)}\right\rangle
$$

which can be alternatively written as,

$$
|\tilde{G}\rangle=|+, 0\rangle+\sum_{N=2,4, . .} a_{N}|+, N\rangle+\sum_{N=1,3, . .} b_{N}|-, N\rangle
$$

The coefficients $a_{N}$ and $b_{N}$ are determined from Eq.(14) and the second order correction to the wave function. The normalized ground state wave function $|\tilde{G}\rangle_{N}$ is

$$
|\tilde{G}\rangle_{N}=\frac{1}{\sqrt{N_{G}}}|\tilde{G}\rangle
$$

where $N_{G}$ is obtained as

$$
N_{G} \equiv\langle\tilde{G} \mid \tilde{G}\rangle=1+\sum_{N=2,4, . .} a_{N}^{2}+\sum_{N=1,3 . .} b_{N}^{2}
$$

Within the modified LF method the ground state wave function for the $d$ oscillators is a displaced Gaussian

$$
\phi(x)=\frac{1}{\pi^{\frac{1}{4}}} \exp \left[-\left(\mathrm{x}-\mathrm{x}_{0}\right)^{2}\right]
$$

where, $x_{0}=-\left(n_{1}-n_{2}\right) \sqrt{2} \lambda$. Including the corrections due to the perturbation the ground state wave function for the $d$ oscillator is obtained as,

$$
\begin{aligned}
G(x) & \equiv \tilde{G}\left(x-x_{0}\right)=\left\langle x-x_{0} \mid 0\right\rangle+\sum_{N=2,4 . .} a_{N}\left\langle x-x_{0} \mid N\right\rangle \\
& +\sum_{N=1,3 \ldots} b_{N}\left\langle x-x_{0} \mid N\right\rangle
\end{aligned}
$$

Note that $G(x)$ and $\tilde{G}(x)$ are the ground state oscillator wave functions for $H_{d}$ and $\tilde{H}_{d}$, respectively. If the electron is located on site 1 then $x_{0}=-\sqrt{2} \lambda$. 
- Correlation function calculation:

The static correlation function $\left\langle n_{1} u_{1}\right\rangle_{0}$ and $\left\langle n_{1} u_{2}\right\rangle_{0}$, where $u_{1}$ and $u_{2}$ are the lattice deformations at site 1 and 2 respectively, produced by an electron at site 1 , indicates the strength of polaron induced lattice deformation and their spread. These correlation functions are determined as

$$
\begin{aligned}
\left\langle n_{1} u_{1}\right\rangle_{0} & =\frac{1}{2}\left[-\left(g_{+}+\lambda\right)+\frac{A_{0}}{N_{G}}\right] \\
\left\langle n_{1} u_{2}\right\rangle_{0} & =\frac{1}{2}\left[-\left(g_{+}-\lambda\right)-\frac{A_{0}}{N_{G}}\right]
\end{aligned}
$$

where

$$
A_{0} \equiv\left\langle\tilde{G}\left|n_{1}\left(d+d^{\dagger}\right)\right| \tilde{G}\right\rangle=\sum_{N=1,3, . .} b_{N}\left[\sqrt{N} a_{N-1}+\sqrt{N+1} a_{N+1}\right]
$$

\section{B. The First Excited State:}

The unperturbed energies of the states $|+, 1\rangle$ and $|-, 0\rangle$ are $\left(\epsilon_{p}+\omega_{0}-t_{e}\left(1-4 \lambda^{2}\right)\right)$ and $\left(\epsilon_{p}+t_{e}\right)$, respectively. For $2 t>\omega_{0}$, the energy of the state $|+, 1\rangle$ is lower than that of $|-, 0\rangle$ for $g_{+}=0$, while it is higher for large values of $g_{+}$when $t_{e}$ becomes negligible. The off-diagonal matrix element of $\tilde{H}_{d}$ between these two states is nonzero. Crossing of the unperturbed energies of these two states at an intermediate value of $g_{+}$and nonzero off-diagonal matrix elements requires that one should follow the degenerate perturbation theory. So, linear combinations of the states $|+, 1\rangle$ and $|-, 0\rangle$ are formed to obtain two new elements of basis states so that $\tilde{H}_{d}$ becomes diagonal in the sub-space spanned by these two states. The first excited state of $\tilde{H}_{d}$ is described by one of the linear combinations which has lower energy. The unperturbed first excited state is given by

$$
\left|\psi_{1}^{(0)}\right\rangle=a|-, 0\rangle+b|+, 1\rangle
$$


The ratio $(c)$ of the coefficients $a$ and $b$ and the unperturbed energy $(\alpha)$ of the first excited state may be found out from the relation

$$
c=\frac{\alpha-H_{11}}{H_{12}}=\frac{H_{12}}{\alpha-H_{22}}
$$

where $H_{11}, H_{22}, H_{12}$ are the matrix elements of $\tilde{H}_{d}$ in the subspace of $|-, 0\rangle$ and $|+, 1\rangle$ and are given in the matrix form in the following,

\begin{tabular}{c|cc}
$\tilde{H}_{d}$ & $|-, 0\rangle$ & $|+, 1\rangle$ \\
\hline$\langle 0,-|$ & $\left(\epsilon_{p}+t_{e}\right)$ & $2 \lambda t_{e}+\omega_{0}\left(g_{+}-\lambda\right)$ \\
$\langle 1,+|$ & $2 \lambda t_{e}+\omega_{0}\left(g_{+}-\lambda\right)$ & $\omega_{0}+\epsilon_{p}-t_{e}\left(1-4 \lambda^{2}\right)$
\end{tabular}

Eq.(23) gives two roots of $\alpha$, the lower value of $\alpha$ (say, $\alpha_{1}$ ) corresponds to the first excited state.

The first order correction to the first excited state wave function is obtained as,

$$
\begin{aligned}
\left|\psi_{1}^{(1)}\right\rangle & =\frac{1}{\sqrt{1+c^{2}}}\left[\sum_{N=2,4, . .} \frac{W_{e}}{\left(\alpha_{1}-E_{-, N}^{(0)}\right)}|-, N\rangle\right. \\
& \left.+\sum_{N=3,5 . .} \frac{W_{o}}{\left(\alpha_{1}-E_{+, N}^{(0)}\right)}|+, N\rangle\right]
\end{aligned}
$$

where $W_{o}=t_{e} \frac{(2 \lambda)^{N}}{\sqrt{N !}}\left(1+2 \lambda c-\frac{c N}{2 \lambda}\right)$

and $W_{e}=W_{o}+\sqrt{2} \omega_{0} c\left(g_{+}-\lambda\right) \delta_{N, 2}$

Second order correction to the first excited state energy is given by,

$$
E_{0}^{(2)}=\frac{1}{1+c^{2}}\left[\sum_{N=2,4, . .} \frac{\left|W_{e}\right|^{2}}{\left(\alpha_{1}-E_{-, N}^{(0)}\right)}+\sum_{N=3,5 . .} \frac{\left|W_{o}\right|^{2}}{\left(\alpha_{1}-E_{+, N}^{(0)}\right)}\right]
$$

\section{Results and discussions}


In this paper we report mainly the results of $t=1.1$ (in a scale of $\omega_{0}=1$ ) for which exact results [4] are available. For the ground state the wave function and the energy have been calculated up to the second order and the third order in perturbation, respectively. For the numerical calculation we consider up to 25 phonon states in the series of Eqs. (14), (15) and (16). It is found that except for very high values of $g_{+}$cosideration of 20 phonon states is more than sufficient, while for large values of $g_{+}(1.8-2.2)$ consideration of 25 phonon states is enough.

In Table-I we have shown the unperturbed energy, the second and third order corrections to the ground state energy. It is seen that the magnitude of the higher order corrections decreases rapidly which clearly indicates the convergence of the series and reasonability of our approach. The second and third order perturbation corrections to the energy are small in both the weak and strong coupling limits and appreciable only in the intermediate coupling limit $1.0 \leq g_{+} \leq 1.3$ where higher order corrections may be necessary.

It may be noted that for lower values of $t$ (results for $t=1.1$ are shown here) the perturbation series converges more rapidly with smaller perturbation corrections. So, the present method based on modified LF transformation is expected to work very satisfactorily for $t<\omega_{0}$.

In Fig. 1 we have shown the single electron energies as a function of $g_{+}$for the ground state (calculated up to the third order) and the first excited state (calculated up to the second order). The results are found to be almost identical with the exact results by Ranninger and Thibblin (within the resolution of Fig. 1 of Ref. 4). It should be mentioned that in a range $1.2<g_{+}<1.4$ the second order correction to the energy for the first excited state is $\sim 10-12 \%$ of the unperturbed energy and so third order correction may be necessary in this region. For other regions the second order correction to the energy of the first excited state is small.

In Fig. 2 we have shown the ground state wavefunction for the $d$ oscillator as 
a function of position $x$ for different values of the e-ph coupling when the electron is located on site 1 . For weak coupling $\left(g_{+}<1\right)$ the wave function shows displaced Gaussian like single peak where the displacement is given by the modified LF value, $x_{0}=-\sqrt{2} \lambda$. However, for $g_{+}=1.3$ an additional prominent shoulder appears. For higher values of $g_{+}$this shoulder takes the form of a broad peak. These results are completely consistent with the results obtained by Ranninger and Thibblin by exact diagonalization study $\llbracket$.

In Fig. 3 we have plotted the variation of the correlation functions $\left\langle n_{1} u_{1}\right\rangle_{0}$ and $\left\langle n_{1} u_{2}\right\rangle_{0}$ with $g_{+}$. Our perturbation results are found to be very close to the exact results of Ref. [4]. It may be mentioned that the second order correction to the ground state wave function becomes very important in determining the shape of the correlation function within our method. In our method the ground state has no component of $|-, 1\rangle$ up to the first order correction, but it appears in the second order correction to the wavefunction. Presence of $|-, 1\rangle$ in the ground state has a significant contribution to the correlation function. The correlation functions are found to be very sensitive to any small correction to the wave function unlike the single elctron energies. We find a slight departure of our results from the exact results of Ref. citeRT at intermediate coupling strength. This is due to the finite series (up to the second order in perturbation for the wave function) that we have considered.

It may be mentioned that recently de Mello and Ranninger concluded from their study of a two site one polaron problem that it would be very difficult for any analytical method to describe the Holstein model except in the extreme adiabatic or nonadiabatic limit. We have shown here that even for $t / \omega_{0}=1.1$ which is in between the above two limits the exact results are fairly reproducible by our analytical method based on the modified LF transformation and perturbation expansion. Comparisons of the energies and the correlation functions in the ground 
state with exact results show that this method, used here, with the second order perturbation in the wave function could match the exact results to a desirable accuracy except in a narrow range of $g_{+}$from 1 to 1.3. For this narrow range of $g_{+}$reasonable results are obtained but one should include higher order corrections to obtain results to match with the exact one. It should be mentioned that as one decreases the value of $t$ and move towards the antiadiabatic limit the convergence becomes better and the region of $g_{+}$, where the perturbation corrections are appreciable, becomes narrower. In that case the perturbation method up to the second order correction in the wave function would describe the system for a wider range of $g_{+}$.

\section{Conclusion}

In the present work we develop an analytical perturbation method within the modified LF approach to deal with an electron-phonon system for the whole range of e-ph coupling strength. This method is applicable from the antiadiabatic limit $\left(t<\omega_{0}\right)$ to the intermediate region of hopping $\left(t \sim \omega_{0}\right)$. Considering a two-site one electron system we have calculated the single electron energies, the ground state oscillator wave functions and the correlation functions (up to the second order perturbation correction to the wave function) and find that the results are in the good agreement with the exact results. The perturbation series converges quite rapidly for all values of $g_{+}$. 
Table I. Variational parameter $(\lambda)$, unperturbed single electron energy (measured with respect to the bare site energy $\epsilon$ ), second order correction and third order correction to the energy for different values of the coupling strength $\left(g_{+}\right)$for the ground state of two-site one polaron problem.

\begin{tabular}{|c|c|c|c|c|}
\hline$g_{+}$ & $\lambda$ & $E_{0}^{(0)}-\epsilon$ & $E_{0}^{(2)}$ & $E_{0}^{(3)}$ \\
\hline 0.2 & .0628 & -1.1125 & -.00007 & .00000 \\
\hline 0.5 & .1619 & -1.1795 & -.00275 & .00001 \\
\hline 0.8 & .2771 & -1.3099 & -.01767 & .00062 \\
\hline 1.0 & .3763 & -1.4397 & -.04564 & .00522 \\
\hline 1.1 & .4421 & -1.5212 & -.07266 & .01408 \\
\hline 1.2 & .5363 & -1.6183 & -.12168 & .03805 \\
\hline 1.3 & 1.0202 & -1.7489 & -.28799 & .09973 \\
\hline 1.4 & 1.3047 & -1.9874 & -.20805 & .02331 \\
\hline 1.7 & 1.6875 & -2.8935 & -.11787 & .00094 \\
\hline 1.9 & 1.8969 & -3.6108 & -.09082 & .00014 \\
\hline 2.2 & 2.1997 & -4.8401 & -.06240 & .00002 \\
\hline
\end{tabular}




\section{References}

[1] T. Holstein, Ann. Phys. (NY) 8, 325 (1959).

[2] A. B. Migdal, Sov. Phys. JETP 7, 996 (1958).

[3] L. G. Lang and Yu A. Firsov, Sov. Phys. JETP 16, 1301 (1963).

[4] J. Ranninger and U. Thibblin, Phys. Rev. B 45, 7730 (1992).

[5] F. Marsiglio, Phys. Letts. A 180, 280 (1993); Physica C 244, 21 (1995).

[6] V. V. Kabanov and D. K. Ray, Phys. Letts. A 186, 438 (1994).

[7] A. S. Alexandrov, V. V. Kabanov and D. K. Ray, Phys. Rev. B 49, 9915 (1994).

[8] E. V. L. de Mello and J. Ranninger, Phys. Rev. B 55, 14872 (1997); Phys. Rev. B 59, 12135 (1999).

[9] A. N. Das and P. Choudhury, Phys. Rev. B 49, 13219 (1994).

[10] P. Choudhury and A. N. Das , Phys. Rev. B 53, 3203 (1996).

[11] A. N. Das and S. Sil, Phyica C 207, 51 (1993); J. Phys.: Condens. Matter 5, 1 (1993).

[12] C. F. Lo and R. Sollie, Phys. Rev. B 45, 7102 (1992).

[13] Yu. A. Firsov and E. K. Kudinov, Phys. Solid. State (AIP) 39 (12), 1930 (1997). 
Figure captions :

FIG. 1. Single electron energies (in units of $\omega_{0}=1$ ) as a function of the coupling strength $\left(g_{+}\right)$. Dashed curve: ground state, solid curve: first excited state.

FIG. 2. Ground state oscillator wave function $G(x)$ as a function of $\mathrm{x}$ for different values of the coupling strength when the electron is located on site 1. Solid: $g_{+}=0.1$, short-dashed: $g_{+}=0.7$, long-dashed: $g_{+}=1.3$ and dot-dashed: $g_{+}=2.0$.

FIG. 3. Plot of the correlation functions (a): $\left\langle n_{1} u_{1}\right\rangle_{0}$ and (b): $\left\langle n_{1} u_{2}\right\rangle_{0}$ versus $g_{+}$. To compare the results of Ref. (4) we use a unit of $\frac{1}{2} \sqrt{\frac{\hbar}{M \omega}}$ for the correlation functions. 